TRANSACTIONS OF THE

AMERICAN MATHEMATICAL SOCIETY

Volume 359, Number 7, July 2007, Pages 3287-3300

S 0002-9947(07)04125-6

Article electronically published on February 21, 2007

\title{
AN ANALOGUE OF THE DESCARTES-EULER FORMULA FOR INFINITE GRAPHS AND HIGUCHI'S CONJECTURE
}

\author{
MATT DEVOS AND BOJAN MOHAR
}

\begin{abstract}
Let $\mathcal{R}$ be a connected 2-manifold without boundary obtained from a (possibly infinite) collection of polygons by identifying them along edges of equal length. Let $V$ be the set of vertices, and for every $v \in V$, let $\kappa(v)$ denote the (Gaussian) curvature of $v: 2 \pi$ minus the sum of incident polygon angles. Descartes showed that $\sum_{v \in V} \kappa(v)=4 \pi$ whenever $\mathcal{R}$ may be realized as the surface of a convex polytope in $\mathbb{R}^{3}$. More generally, if $\mathcal{R}$ is made of finitely many polygons, Euler's formula is equivalent to the equation $\sum_{v \in V} \kappa(v)=$ $2 \pi \chi(\mathcal{R})$ where $\chi(\mathcal{R})$ is the Euler characteristic of $\mathcal{R}$. Our main theorem shows that whenever $\sum_{v \in V: \kappa(v)<0} \kappa(v)$ converges and there is a positive lower bound on the distance between any pair of vertices in $\mathcal{R}$, there exists a compact closed 2 -manifold $\mathcal{S}$ and an integer $t$ so that $\mathcal{R}$ is homeomorphic to $\mathcal{S}$ minus $t$ points, and further $\sum_{v \in V} \kappa(v) \leq 2 \pi \chi(\mathcal{S})-2 \pi t$.

In the special case when every polygon is regular of side length one and $\kappa(v)>0$ for every vertex $v$, we apply our main theorem to deduce that $\mathcal{R}$ is made of finitely many polygons and is homeomorphic to either the 2 -sphere or to the projective plane. Further, we show that unless $\mathcal{R}$ is a prism, antiprism, or the projective planar analogue of one of these that $|V| \leq 3444$. This resolves a recent conjecture of Higuchi.
\end{abstract}

\section{INTRODUCTION}

A polygonal surface $\mathcal{R}$ is a simply connected 2-manifold without boundary which is obtained from a (possibly infinite) collection of disjoint simple polygons in $\mathbb{R}^{2}$ by identifying them along edges of equal length. Based on this construction, $\mathcal{R}$ may be viewed as an embedded graph, and accordingly, we equip it with three distinguished sets: vertices, edges, and faces, respectively denoted $V(\mathcal{R}), E(\mathcal{R})$, and $F(\mathcal{R})$, and defined to be the set of all subsets of $\mathcal{R}$ which correspond (respectively) to a vertex of a polygon, edge of a polygon, or polygon itself. We view vertices, edges, and faces both combinatorially and as subsets of $\mathcal{R}$; two such objects are defined to be incident if one is a proper subset of the other. The space $\mathcal{R}$ is also equipped with a natural intrinsic metric: the distance between two points is the length of the shortest rectifiable curve joining them. This metric gives each point $x \in \mathcal{R}$ a Gaussian curvature which we denote by $\kappa(x)$. If $x$ is not a vertex, then $\kappa(x)=0$. If $x$ is a vertex, then $\kappa(x)$ is equal to $2 \pi$ minus the sum over all faces $f$ incident

Received by the editors July 2, 2004 and, in revised form, May 11, 2005.

2000 Mathematics Subject Classification. Primary 05C10.

The first author was supported in part by the SLO-USA Grant BI-US/04-05/36 and by the Slovenian grant L1-5014.

The second author was supported in part by the Ministry of Education, Science and Sport of Slovenia, Research Program P1-0297 and Research Project J1-6150.

(C)2007 American Mathematical Society Reverts to public domain 28 years from publication 
with $x$ of the angle of $f$ at $x$ (if a face is incident with $v$ more than once, then all incident angles count). Descartes (see, e.g., [2]) proved the following important theorem regarding the curvature of vertices in a 3-polytope.

Theorem 1.1 (Descartes). If $\mathcal{R}$ is a polygonal surface which may be realized as the surface of a convex polytope in $\mathbb{R}^{3}$, then

$$
\sum_{v \in V(\mathcal{R})} \kappa(v)=4 \pi .
$$

We define the polygonal surface $\mathcal{R}$ to be finite if $F(\mathcal{R})$ is finite. For finite polygonal surfaces, the Gauss-Bonnet formula gives us a curvature identity which is a generalization of the Descartes theorem. In fact, this generalization is equivalent to Euler's formula and may be stated as follows (here we let $\chi(\mathcal{S})$ denote the Euler characteristic of a surface $\mathcal{S}$ ).

Theorem 1.2 (Euler-Gauss-Bonnet formula). If $\mathcal{R}$ is a finite polygonal surface, then

$$
\sum_{v \in V(\mathcal{R})} \kappa(v)=2 \pi \chi(\mathcal{R}) .
$$

Our main theorem, stated below, gives a related formula which holds for infinite polygonal metric spaces. For that, a technical condition, which assures that the metric space $\mathcal{R}$ is complete, is needed. We define $\nu=\nu(\mathcal{R})$ to be the infimum of all distances between distinct vertices, and we say that $\mathcal{R}$ is tame if $\nu>0$.

Theorem 1.3. If $\mathcal{R}$ is a tame polygonal surface and $\sum_{v \in V(\mathcal{R}): \kappa(v)<0} \kappa(v)$ converges, then there exists a compact closed 2-manifold $\mathcal{S}$ and an integer $t$ so that $\mathcal{R}$ is homeomorphic to $\mathcal{S}$ minus $t$ points, and further

$$
\sum_{v \in V(\mathcal{R})} \kappa(v) \leq 2 \pi \chi(\mathcal{S})-2 \pi t .
$$

For instance, if $\kappa(v)$ is everywhere non-negative, and takes a positive value on at least one vertex, then the above theorem implies that either $\mathcal{R}$ is homeomorphic to the plane, or $\mathcal{R}$ is finite and homeomorphic to either the sphere or the projective plane.

A relatively simple example shows that the above theorem does not hold without the assumption that $\mathcal{R}$ is tame. Let $\mathcal{S}$ be the surface obtained from the unit sphere $S^{2}$ in $\mathbb{R}^{3}$ by removing a finite set of at least three points. If $\mathcal{T}$ is a triangulation of $\mathcal{S}$ with all edges being geodesic segments in $S^{2}$, then $\mathcal{T}$ gives rise to a polygonal surface $\mathcal{T}^{\prime}$ whose vertices are the vertices of $\mathcal{T}$ and edges are the corresponding straight line segments in $\mathbb{R}^{3}$. Now the Gaussian curvature at every vertex is positive, and it follows that the inequality of Theorem 1.3 cannot hold.

An interesting special case is when each face of $\mathcal{R}$ is a regular polygon of side length one. In this case, the space $\mathcal{R}$ may be described by an embedded graph, and several authors have used this connection to define a combinatorial notion of curvature for an embedded graph. We offer a definition below which is identical to one made by Stone [9], 10], Woess [12] and Higuchi [4, and is equivalent to one given by Gromov [3].

For any graph $G$, let $\operatorname{deg}(v)$ denote the degree of a vertex $v \in V(G)$. If $G$ is embedded in a surface, and $f$ is a face, we let $\operatorname{deg}(f)$ denote the size of $f$ - which 
we shall also call its degree. We define the combinatorial curvature of the vertex $v \in V(G)$ to be

$$
\phi(v)=1-\frac{\operatorname{deg}(v)}{2}+\sum_{f \sim v} \frac{1}{\operatorname{deg}(f)}
$$

where the sum is taken over all faces $f$ incident with $v$, counted according to multiplicity of incidences. As suggested above, the motivation for this definition is that replacing each face of $G$ by a regular polygon of side length one yields a polygonal surface for which $\kappa(v)=2 \pi \phi(v)$ for every vertex $v$. By way of this correspondence, our main theorem has a natural corollary for combinatorial curvature which we offer after introducing some added terminology.

A one-way infinite path in a graph $G$ is called a ray. Two rays $A$ and $B$ are equivalent if for every finite set $X$ of vertices of $G$, the (unique) component of $G-X$ containing an infinite number of vertices in $A$ is also the (unique) component of $G-X$ containing an infinite number of vertices in $B$. It is straightforward to verify that this relation is an equivalence relation. Equivalence classes of rays in $G$ are called $e n d s$, and we let $\operatorname{Ends}(G)$ denote the set of ends of the graph $G$.

Corollary 1.4. Let $G$ be a graph that is 2-cell embedded in the surface $\mathcal{S}$ so that each face has finite size and let $t=|\operatorname{Ends}(G)|$. If $G$ has only finitely many vertices $v$ with $\phi(v)<0$, then there exists a compact closed 2-manifold $\mathcal{T}$ so that $\mathcal{S}$ is homeomorphic to $\mathcal{T}$ minus $t$ points, and further

$$
\sum_{v \in V(G)} \phi(v) \leq \chi(\mathcal{T})-t
$$

If all faces of an embedded graph have the same size, the combinatorial curvature of a vertex may be expressed in terms of its degree. Such families provide a natural setting for the above result. For instance, applying this result to triangulations yields the following.

Corollary 1.5. Let $G$ be a graph which is a triangulation of the surface $\mathcal{S}$ and let $t=|\operatorname{Ends}(G)|$. If $G$ has only finitely many vertices of degree at least 7 , then there exists a compact closed 2-manifold $\mathcal{T}$ so that $\mathcal{S}$ is homeomorphic to $\mathcal{T}$ minus $t$ points, and further

$$
\sum_{v \in V(G)}(6-\operatorname{deg}(v)) \leq 6 \chi(\mathcal{T})-6 t .
$$

It is fairly easy to find families of triangulations for which the above bound is tight. Begin with a triangulation $G$ of a compact closed 2-manifold and a facial triangle $f$ with vertices $x_{1}, x_{2}, x_{3}$ (and note that $G$ is tight with respect to the above inequality). Next, draw an infinite sequence of nested triangles in $f$ converging to a point $z$ which we remove from the surface, and add edges between these nested triangles so that all newly created vertices are of degree six and so that the sum of the degrees of $x_{1}, x_{2}$, and $x_{3}$ increases by six. This newly formed graph is tight with respect to the above inequality, and by repeating this construction, we may form tight instances for any surface and any number of ends of the graph.

In a recent paper [4, Higuchi made a conjecture equivalent to the one given below concerning graphs with everywhere positive combinatorial curvature.

Conjecture 1.6 ([4, Higuchi]). Let $G$ be a graph which is 2-cell embedded in a surface $\mathcal{S}$ so that every vertex and face has degree $\geq 3$. If $\mathcal{S}$ is homeomorphic to a subset of the 2-sphere and $\phi$ is everywhere positive, then $G$ is finite. 
A special case of this conjecture when $G$ is 3-regular was recently resolved by Sun and $\mathrm{Yu}$ [11]. Using Theorem 1.3 we prove the following result, which resolves Higuchi's conjecture in full.
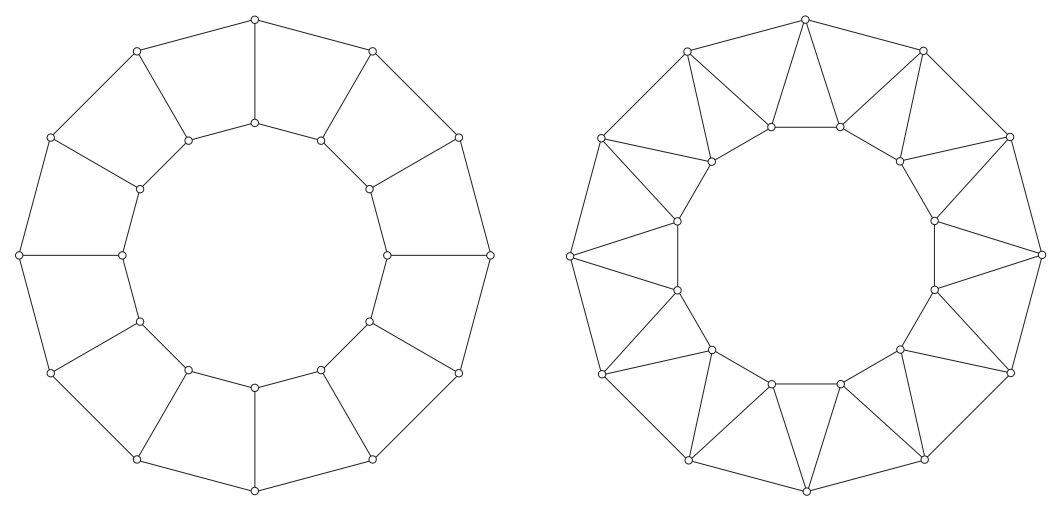

FIgURE 1. Examples of a prism and an antiprism

Theorem 1.7. Let $G$ be a graph which is 2-cell embedded in a surface $\mathcal{S}$ so that every vertex and face has degree $\geq 3$. If $\phi$ is everywhere positive, then $\mathcal{S}$ is homeomorphic to either the 2-sphere or the projective plane and $G$ is finite. Furthermore, if $G$ is not a prism, antiprism, or the projective planar analogue of one of these, then $|V(G)| \leq 3444$.
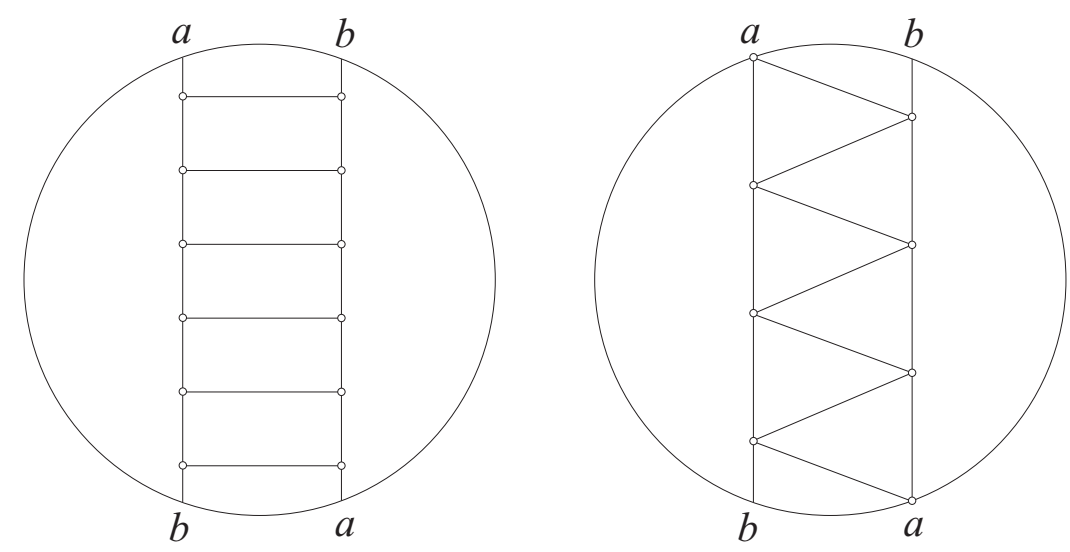

Figure 2. Projective planar prism and antiprism

Examples of a prism and an antiprism (planar and projective planar) are shown in Figures 1 and 2. The projective planar prisms are also known as Möbius ladder graphs.

It would be interesting to find the smallest number $C$ which could take the place of 3444 in the above theorem. A lower bound of 120 is given by the great rhombic icosidodecahedron. This bound has been improved by Réti et al. 8], who found a 3-connected planar graph (which is not a prism or antiprism) with 
positive combinatorial curvature and 138 vertices. Thus $138 \leq C \leq 3444$. Although little effort has been made here to optimize our upper bound, it appears that a considerably more detailed analysis would be required to get close to the correct value for $C$.

Although all of our results concern polygonal metric spaces, many of these results can be extended to more general spaces by way of approximation. As noted by Higuchi, Theorem 1.7 can be viewed as a discrete analogue of a result of Myers 7] who proved that every complete Riemannian manifold with Ricci curvature bounded below by a positive constant $\kappa_{0}$ is compact, has volume bounded in terms of $\kappa_{0}$ and has finite fundamental group. On the other hand, Theorem 1.3 shows other possible directions for improvements of such results. One such extension may be a conjecture of Milnor [5] that every complete Riemannian manifold with non-negative Ricci curvature has finitely generated fundamental group.

\section{Preliminaries}

In this section we shall introduce the main terms and objects we will use in the remainder of the paper. Many of these terms are standard in the context of polygonal surfaces. For a good introduction to this subject, we recommend [1].

In a polygonal surface $\mathcal{R}$, the distance function gives rise to a length function $\ell$ which assigns length to any rectifiable curve in $\mathcal{R}$. A geodesic is an $\operatorname{arc} \alpha \subseteq \mathcal{R}$ which is locally length minimizing. We think of a geodesic as having no direction, and if $x, y \in \mathcal{R}$ are the ends of $\alpha$, then we say that $\alpha$ joins $x$ and $y$. A geodesic is nice if both of its ends are vertices and no interior point of $\alpha$ is a vertex.

The distance function also gives rise to a concept of angle. If $x$ is a point in the boundary of the set $\mathcal{T} \subseteq \mathcal{R}$ and there exists a neighborhood $N$ of $x$ so that the intersection of the boundary of $\mathcal{T}$ and $N$ is a rectifiable curve containing $x$, then we call $\mathcal{T}$ a half-neighborhood of $x$ and we let $\angle(\mathcal{T}, x)$ denote the angle of $\mathcal{T}$ at $x$. The inner boundary curvature of $\mathcal{T}$ at $x$ is defined to be $\iota(\mathcal{T}, x)=\pi-\angle(\mathcal{T}, x)$. If $\alpha$ is a simple curve containing $x$ in its interior, and there is a neighborhood $N$ of $x$ so that $\alpha$ and the boundary of $\mathcal{T}$ coincide inside $N$, then we call $\mathcal{T}$ a half-neighborhood of $x$ along $\alpha$. Notice that if $\mathcal{T}_{1}$ and $\mathcal{T}_{2}$ are disjoint half-neighborhoods of $x$ along $\alpha$, then $\iota\left(\mathcal{T}_{1}, x\right)+\iota\left(\mathcal{T}_{2}, x\right)=\kappa(x)$. Every point in a polygonal surface has a neighborhood which is isometric to the lateral surface of a truncated polyhedral cone. It follows immediately from this that whenever a simple geodesic passes through a point $x$, every half neighborhood $\mathcal{T}$ of $x$ along this geodesic must satisfy $\angle(\mathcal{T}, x) \geq \pi$ (so in particular, a geodesic cannot pass through a vertex $v$ unless $\kappa(v) \leq 0$ ). More generally, this property can be used to characterize geodesics. Since this observation will be used frequently, we have sumarized it below.

Observation 2.1. Let $\alpha$ be a simple rectifiable curve in a polygonal surface $\mathcal{R}$. Then the following statements are equivalent:

(i) $\alpha$ is a geodesic.

(ii) For every $x$ in the interior of $\alpha$ and every half-neighborhood $\mathcal{T}$ of $x$ along $\alpha$, we have $\angle(\mathcal{T}, x) \geq \pi$.

(iii) For every face $f$, every subarc of $\alpha$ contained in $f$ is a line segment. For every point $x$ in the interior of $\alpha$ which is not in the interior of a face and every half-neighborhood $\mathcal{T}$ of $x$ along $\alpha$, we have $\angle(\mathcal{T}, x)=\pi$ if $x$ is in the interior of an edge and $\angle(\mathcal{T}, x) \geq \pi$ if $x$ is a vertex. 
In particular, the above observation shows that every edge is a geodesic.

A geodesic walk $W$ is a sequence $x_{0}, \alpha_{1}, x_{1}, \alpha_{2}, \ldots, x_{k-1}, \alpha_{k}, x_{k}$ where every $x_{i}$ is a point in $\mathcal{R}$, every $\alpha_{i}$ is a geodesic with ends $x_{i-1}$ and $x_{i}$, and the points $x_{i-1}$ and $x_{i}$ are distinct whenever $1 \leq i \leq k$. Every $x_{i}$ is called a corner of $W$, and the sequence $x_{0}, x_{1}, \ldots, x_{k}$ is called the sequence of corners. We say that $W$ is closed if $x_{0}=x_{k}$, and we say that $W$ is simple if every $\alpha_{i}$ is simple, any two geodesics $\alpha_{i}$ and $\alpha_{j}(i \neq j)$ intersect only at their ends, and either $W$ is closed and $x_{0}, \ldots, x_{k-1}$ are distinct, or $W$ is not closed and $x_{0}, \ldots, x_{k}$ are distinct. We say that $W$ is nice if every geodesic $\alpha_{i}$ is nice. The length of $W$ is $\ell(W)=\sum_{i=1}^{k} \ell\left(\alpha_{i}\right)$ and the combinatorial length of $W$ is $\lambda(W)=k$. Note that the combinatorial length is in general unrelated to the (ordinary) length. For convenience of notation, we shall associate the walk $W$ with the subset $\bigcup_{i=1}^{k} \alpha_{i} \subseteq \mathcal{R}$.

For any subset $\mathcal{S} \subseteq \mathcal{R}$, we let $V^{i}(\mathcal{S})$ denote the set of vertices in the interior of $\mathcal{S}$ and $V^{b}(\mathcal{S})$ the set of vertices in the boundary of $\mathcal{S}$. The following basic observation is quite useful, since it permits us to reason about certain triangles using plane geometry. The (easy) proof follows by cutting the surface along the geodesics $\alpha_{1}$, $\alpha_{2}$, and $\alpha_{3}$.

Observation 2.2. Let $x_{0}, \alpha_{1}, x_{1}, \alpha_{2}, x_{2}, \alpha_{3}, x_{0}$ be a nice closed geodesic walk which bounds a disc $D$ with $V^{i}(D)=\emptyset$. Then there exist three points $A, B, C$ in $\mathbb{R}^{2}$ so that the triangle $A B C$ is congruent to $D$ (i.e. $\overline{A B}=\ell\left(\alpha_{1}\right), \angle C A B=\angle\left(D, x_{0}\right)$, etc.).

Next we introduce an object which will be the main subject for much of the rest of this article. If $(s, t, u)$ is a triple of non-negative integers, then an $(s, t, u)$-front is a subset $\mathcal{Q} \subseteq \mathcal{R}$ with the following properties:

(i) There exists a surface $\mathcal{S}$ of Euler characteristic $s$ so that $\mathcal{Q}$ is homeomorphic to the space obtained from $\mathcal{S}$ by removing $t$ disjoint open discs. (In particular, $\mathcal{Q}$ is closed.)

(ii) The boundary of $\mathcal{Q}$ can be written as a union of nice simple closed geodesic walks $W_{1}, \ldots, W_{t}$, called boundary walks, so that the intersection of any two such walks is a subset of $V(\mathcal{R})$.

(iii) There are exactly $u$ path-connected components of $\mathcal{R} \backslash \mathcal{Q}$.

(iv) The set $V^{i}(\mathcal{Q})$ is finite.

Extending some earlier definitions, we define the length of a front $\mathcal{Q}$ to be $\ell(\mathcal{Q})=$ $\sum_{i=1}^{t} \ell\left(W_{i}\right)$ and the combinatorial length of $\mathcal{Q}$ to be $\lambda(\mathcal{Q})=\sum_{i=1}^{t} \lambda\left(W_{i}\right)$. For $x \in$ $V^{b}(\mathcal{Q})$, let $J=\left\{j \mid 1 \leq j \leq k, x \in W_{j}\right\}$, and for every $j \in J$, let $\mathcal{T}_{j}$ be an open halfneighborhood of $x$ along $W_{i}$ which is disjoint from $\mathcal{Q}$. We define the total angle of $\mathcal{Q}$ at $x$ to be $\angle(\mathcal{Q}, x)=2 \pi-\kappa(x)-\sum_{j \in J} \angle\left(\mathcal{T}_{j}, x\right)$, and we define the inner boundary curvature of $\mathcal{Q}$ at $x$ to be $\iota(\mathcal{Q}, x)=\pi(2-|J|)-\angle(\mathcal{Q}, x)=\kappa(x)-\sum_{j \in J} \iota\left(\mathcal{T}_{j}, x\right)$. The following useful identity follows from these definitions:

$$
\sum_{x \in V^{b}(\mathcal{Q})} \iota(\mathcal{Q}, x)=2 \pi\left|V^{b}(\mathcal{Q})\right|-\pi \lambda(\mathcal{Q})-\sum_{x \in V^{b}(\mathcal{Q})} \angle(\mathcal{Q}, x) .
$$

\section{The Gauss-Bonnet formula}

The Gauss-Bonnet formula relates the Gaussian curvature in the interior of a surface to the curvature of the boundary. We require a version of this result for the special case of polygonal metric spaces, which we will state in terms of fronts. In 
this special case, the Gauss-Bonnet formula may be deduced from Euler's formula, and for the sake of completeness, we include its derivation.

Theorem 3.1 (Gauss-Bonnet). If $\mathcal{Q}$ is an $(s, t, u)$-front in $\mathcal{R}$, then

$$
\sum_{x \in V^{i}(\mathcal{Q})} \kappa(x)+\sum_{y \in V^{b}(\mathcal{Q})} \iota(\mathcal{Q}, y)=2 \pi s-2 \pi t .
$$

Proof. We shall modify our polygonal surface $\mathcal{R}$ to form a new polygonal surface $\mathcal{R}^{\prime}$ by introducing new edges and vertices (and thus faces) without changing the structure of the metric space. First, at each point $x$ in the boundary of $\mathcal{Q}$ through which an edge passes from $\mathcal{Q}$ to $\mathcal{R} \backslash \mathcal{Q}$, subdivide this edge by placing a new vertex at $x$. Then, whenever $x$ and $y$ are consecutive vertices on the boundary of $\mathcal{Q}$, we add an edge from $x$ to $y$ along this stretch of the boundary if such an edge does not already exist. Note that only finitely many new vertices are added in this process, since there are only finitely many vertices in $\mathcal{Q}$, each of finite degree. Now, $\mathcal{Q}$ is a front of $\mathcal{R}^{\prime}$ with the special property that all points in the boundary of $\mathcal{Q}$ lie in $V\left(\mathcal{R}^{\prime}\right) \cup E\left(\mathcal{R}^{\prime}\right)$. Furthermore, every new vertex $x$ introduced on the boundary of $\mathcal{Q}$ has $\iota(\mathcal{Q}, x)=0$, so to prove the above result, it suffices to prove it for $\mathcal{Q}$ considered as a front in the space $\mathcal{R}^{\prime}$. Let $H$ be the embedded graph consisting of all vertices and edges of $\mathcal{R}^{\prime}$ which lie in $\mathcal{Q}$. By construction, $H$ is 2-cell embedded in $\mathcal{Q}$ and each boundary component of $\mathcal{Q}$ is bounded by a cycle of $H$. The following equation relies on the identity at the close of the previous section, the observation that the sum of the interior angles of a plane $k$-gon is $\pi(k-2)$, and Euler's formula applied to the graph $H$ :

$$
\begin{aligned}
\sum_{x \in V^{i}(\mathcal{Q})} \kappa(x)+\sum_{y \in V^{b}(\mathcal{Q})} \iota(\mathcal{Q}, y) & =2 \pi|V(H)|-\pi \lambda(\mathcal{Q})-\pi \sum_{f \in F(H)}(\operatorname{deg}(f)-2) \\
& =2 \pi(|V(H)|-|E(H)|+|F(H)|) \\
& =2 \pi s-2 \pi t .
\end{aligned}
$$

This completes the proof.

The Descartes-Euler formula may be viewed as a corollary of this theorem applied to the front $\mathcal{Q}=\mathcal{R}$.

Let $\mathcal{Q}$ be an $(s, t, u)$-front with $t>0$. We define the value $\Theta(\mathcal{Q})$ by the following rule:

$$
\Theta(\mathcal{Q})=\frac{\nu}{\ell(\mathcal{Q})}\left(\sum_{x \in V^{i}(\mathcal{Q})} \kappa(x)-2 \pi s+2 \pi u+\sum_{y \in V(\mathcal{R}) \backslash V^{i}(Q): \kappa(y)<0} \kappa(y)\right)
$$

where $\nu$ is the infimum of all distances among distinct vertices of $\mathcal{R}$. The motivation for this definition is the following corollary, which is all we shall require from this section in the sequel.

Corollary 3.2. If $\mathcal{Q}$ is a non-empty front in $\mathcal{R}$ with $\Theta(\mathcal{Q}) \geq 0$, then there exists a boundary walk $W$ of $\mathcal{Q}$, a vertex $x \in W$, and an open half-neighborhood $\mathcal{T}$ of $x$ along $W$ which is disjoint from $\mathcal{Q}$ so that $\iota(\mathcal{T}, x) \geq \Theta(\mathcal{Q})$.

Proof. We shall assume that $\mathcal{Q}$ is an $(s, t, u)$-front. Let $W_{1}, \ldots, W_{t}$ be the boundary walks of $\mathcal{Q}$ and for $1 \leq i \leq t$, let $\mathcal{T}_{i}$ be an open subset of $\mathcal{R} \backslash \mathcal{Q}$ which is a halfneighborhood of every vertex in $W_{i}$ along $W_{i}$. For convenience, we will further assume that $V^{b}\left(\mathcal{T}_{i}\right)$ is precisely the vertex set of $W_{i}$. This brings us to the following 
inequality (in which the second line follows from the Gauss-Bonnet formula and the observation that $t \geq u$ ):

$$
\begin{aligned}
\sum_{i=1}^{t} \sum_{x \in V^{b}\left(\mathcal{T}_{i}\right)} \iota\left(\mathcal{T}_{i}, x\right) & =\sum_{x \in V^{b}(\mathcal{Q})}(\kappa(x)-\iota(\mathcal{Q}, x)) \\
& \geq \frac{\ell(\mathcal{Q})}{\nu} \Theta(\mathcal{Q}) \\
& \geq \lambda(\mathcal{Q}) \Theta(\mathcal{Q}) .
\end{aligned}
$$

Since there are exactly $\lambda(\mathcal{Q})$ pairs $\left(x, \mathcal{T}_{i}\right)$ with $x \in V^{b}\left(\mathcal{T}_{i}\right)$, it follows by averaging that we may choose a pair with $\iota\left(\mathcal{T}_{i}, x\right) \geq \Theta(\mathcal{Q})$, as desired.

\section{Some Plane GeOMEtRY}

In this section we prove an easy but essential inequality from plane geometry which will be used later.

Lemma 4.1. Let $X, Y, Z, W$ be points in the plane, and assume that $W$ lies in the triangle $X Y Z$ and that $W$ is at distance at least d from $Y$. Let $a, b, c, e, f$ be the respective lengths of the line segments $X Y, Y Z, X Z, X W, W Z$, and let $\theta$ be the angle $X Y Z$. If $a, b \geq d$, then

(a) $(a+b)-(e+f) \geq 2 d\left(1-\sin \frac{\theta}{2}\right)$.

(b) $a+b-c \geq 2 d\left(1-\sin \frac{\theta}{2}\right)$.

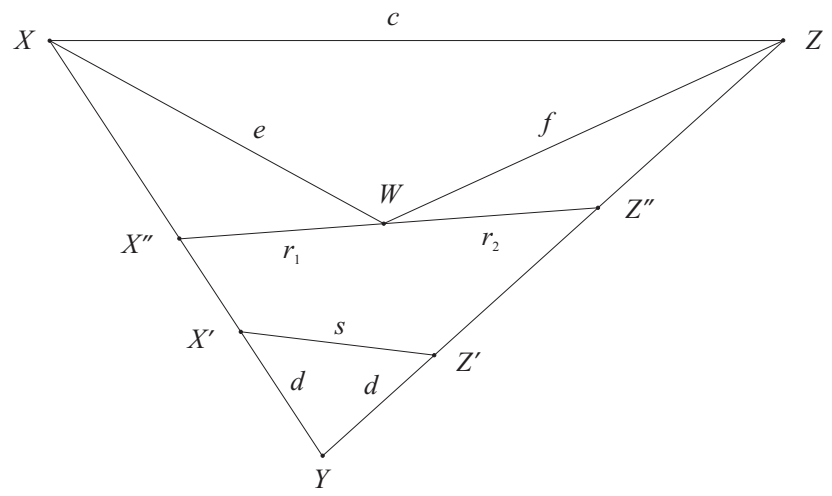

Figure 3. A plane geometry figure

Proof. Let $X^{\prime}$ and $Z^{\prime}$ be the points at distance $d$ from $Y$ which are, respectively, on the line segments $Y X$ and $Y Z$. Now, the line through $X^{\prime}$ and $Z^{\prime}$ separates $Y$ and $W$, so we may choose points $X^{\prime \prime}$ on $X^{\prime} X$ and $Z^{\prime \prime}$ on $Z^{\prime} Z$ so that $W$ lies on the line segment $X^{\prime \prime} Z^{\prime \prime}$. Let $a^{\prime \prime}, b^{\prime \prime}, r_{1}, r_{2}, s$ denote the respective lengths of the line segments $Y X^{\prime \prime}, Y Z^{\prime \prime}, X^{\prime \prime} W, Z^{\prime \prime} W$, and $X^{\prime} Z^{\prime}$. Now we have $s=2 d \sin \frac{\theta}{2}$. By repeated applications of the triangular inequality we find

$$
\begin{aligned}
a+b & =\left(a^{\prime \prime}+b^{\prime \prime}\right)-\left(r_{1}+r_{2}\right)+\left(a-a^{\prime \prime}+r_{1}\right)+\left(b-b^{\prime \prime}+r_{2}\right) \\
& \geq\left(a^{\prime \prime}+b^{\prime \prime}\right)-\left(r_{1}+r_{2}\right)+e+f \\
& =(2 d-s)+\left(\left(a^{\prime \prime}-d\right)+\left(b^{\prime \prime}-d\right)+s\right)-\left(r_{1}+r_{2}\right)+e+f \\
& \geq 2 d\left(1-\sin \frac{\theta}{2}\right)+e+f
\end{aligned}
$$


which yields (a). The inequality (b) is just a special case of (a) when $W=X$. This completes the proof.

\section{THE MAIN THEOREM}

The main theorem requires a few preliminary lemmas. Our first observation and first lemma are technical arguments which show the existence of geodesic walks with useful properties. Throughout this section we shall assume that $\mathcal{R}$ is a tame polygonal surface. If $W$ is a geodesic or a simple geodesic walk and $x, y$ are points in $W$, then we let $W[x, y]$ denote the walk or geodesic obtained by restricting $W$ to the interval between $x$ and $y$.

Observation 5.1. Let $W$ be a geodesic walk in $\mathcal{R}$ given by $u, \alpha_{1}, v, \alpha_{2}, w$, let $\mathcal{T}$ be a half-neighborhood of $v$ along $W$, and assume that $\angle(\mathcal{T}, v)<\pi$. Then there exists a point $q$ on $\alpha_{1}$ and a geodesic walk $W^{\prime}$ from $w$ to $q$ so that the concatenation of $q, \alpha_{1}[q, v], v, \alpha_{2}, w$ and $W^{\prime}$ bounds a disc $\mathcal{D}$ with the following properties:

(1) $\mathcal{D}$ has no vertices in its interior.

(2) There exists a half-neighborhood $\mathcal{T}^{\prime}$ of $v$ with $\mathcal{T}^{\prime} \subseteq \mathcal{T} \cap \mathcal{D}$.

(3) $\iota(\mathcal{D}, z)=0$ for every $z$ in the interior of $W^{\prime}$.

(4) Either $q=u$ or there exists a vertex in the interior of $W^{\prime}$.

Proof. Say that a point $t$ in $\alpha_{1}$ is good if there exists a geodesic walk $W^{\prime}$ from $w$ to $t$ so that the concatenation of $t, \alpha_{1}[t, v], v, \alpha_{2}, w$ and $W^{\prime}$ bounds a disc $\mathcal{D}$ which satisfies properties (1)-(3) above. It follows from the assumption that $\mathcal{R}$ is tame and Observation 2.1 that there exists a good point. Further, the set of all good points together with $v$ form a geodesic of the form $\alpha_{1}[q, v]$, and the walk $W^{\prime}$ and $\operatorname{disc} \mathcal{D}$ for the point $q$ satisfy (1)-(4) above.

Lemma 5.2. Let $W$ be a nice geodesic walk in $\mathcal{R}$ given by $u, \alpha_{1}, v, \alpha_{2}, w$, let $\mathcal{T}$ be a half-neighborhood of $v$ along $W$, and assume that $\angle(\mathcal{T}, v)<\pi$. Then there exists a nice geodesic walk $W^{\prime}$ from $w$ to $u$ so that the concatenation of $W$ and $W^{\prime}$ bounds a disc $\mathcal{D}$ which satisfies the following properties:

(1) $\iota(\mathcal{D}, x) \leq 0$ for every vertex $x$ in the interior of $W^{\prime}$.

(2) There exists a half-neighborhood $\mathcal{T}^{\prime}$ of $v$ with $\mathcal{T}^{\prime} \subseteq \mathcal{T} \cap \mathcal{D}$.

(3) $\ell\left(W^{\prime}\right) \leq \ell(W)$.

Proof. We shall form $W^{\prime}$ in stages by the following procedure. Initially, set $v_{0}=v$, $w_{0}=w, \beta_{0}=\alpha_{2}$, and set $W^{\prime}$ to be the one vertex walk given by the sequence $w$. For step $i$, we apply Observation 5.1 to the walk $u, \alpha_{1}\left[u, v_{i-1}\right], v_{i-1}, \beta_{i-1}, w_{i-1}$ to choose a point $v_{i}$ on $\alpha_{1}\left[u, v_{i-1}\right]$ and a walk $W_{i}$ from $w_{i-1}$ to $v_{i}$. If $v_{i}=u$, then update $W^{\prime}$ by concatenating it with $W_{i}$, and stop. If $v_{i} \neq u$, then let $w_{i}$ be the vertex on $W_{i}$ which is closest (along this walk) to $v_{i}$, set $\beta_{i}$ to be the geodesic $W_{i}\left[w_{i}, v_{i}\right]$, update $W^{\prime}$ by concatenating it with $W_{i}\left[w_{i-1}, w_{i}\right]$, and pass to the next step. If our procedure does not terminate until after step $i$, then by the triangle inequality, we have

$$
\begin{aligned}
\ell\left(\alpha_{1}\left[u, v_{i}\right]\right)+\ell\left(\beta_{i}\right)+\nu & \leq \ell\left(\alpha_{1}\left[u, v_{i}\right]\right)+\ell\left(W_{i}\right) \\
& \leq \ell\left(\alpha_{1}\left[u, v_{i-1}\right]\right)+\ell\left(\beta_{i-1}\right)
\end{aligned}
$$

so it follows that our procedure always terminates. An easy induction shows that the resulting walk satisfies properties (1), (2), and (3) above. 
For any (positive) real number $\zeta$ and any walk $W$, we define the $\zeta$-weight of $W$ to be $w_{\zeta}(W)=\zeta^{-1} \ell(W)-\lambda(W)$. Similarly, define the $\zeta$-weight of a front $\mathcal{Q}$ to be $w_{\zeta}(\mathcal{Q})=\zeta^{-1} \ell(\mathcal{Q})-\lambda(\mathcal{Q})$. We define the front $\mathcal{Q}$ to be full if every connected component of $\mathcal{R} \backslash \mathcal{Q}$ contains infinitely many vertices.

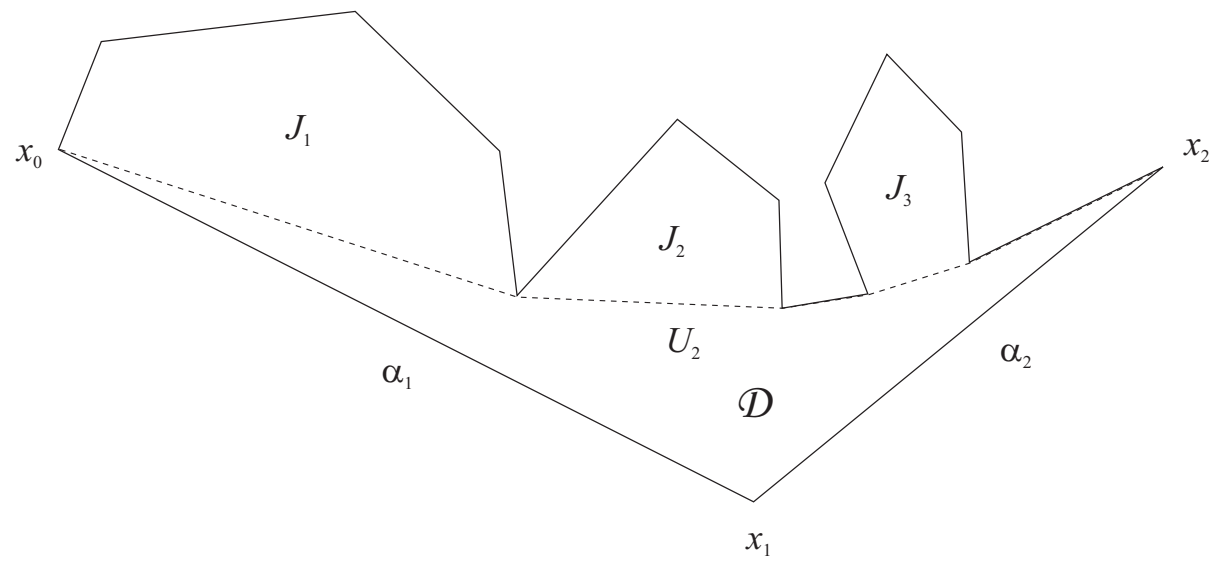

Figure 4. Change of the boundary walk $W$

Lemma 5.3. If $\sum_{v \in V(\mathcal{R}): \kappa(v)<0} \kappa(v)$ converges and $V(\mathcal{R})$ is infinite, then $\Theta(\mathcal{Q}) \leq$ 0 for every full front $\mathcal{Q}$.

Proof. We suppose (for a contradiction) that $\mathcal{Q}_{0}$ is a full front and $\Theta\left(\mathcal{Q}_{0}\right)=\theta_{0}>0$. Set $\zeta=2 \nu\left(1-\cos \frac{\theta_{0}}{2}\right)=2 \nu\left(1-\sin \frac{\pi-\theta_{0}}{2}\right)$. It follows from Corollary 3.2 that $\theta_{0}<\pi$, so we have $0<\zeta<\nu$. It follows from this that the $\zeta$-weight of any front is nonnegative. Now, choose a full $(s, t, u)$-front $\mathcal{Q}$ with the following properties:

(1) $\Theta(\mathcal{Q}) \geq \theta_{0}$

(2) $\left\lceil w_{\zeta}(\mathcal{Q})\right\rceil$ is minimum subject to $(1)$.

It follows from Corollary 3.2 that we may choose a boundary walk $W$ of $\mathcal{Q}$ with sequence $x_{0}, \alpha_{1}, x_{1}, \ldots, x_{k}$, and an open half-neighborhood $\mathcal{T}$ of $x_{1}$ along $W$ so that $\mathcal{T} \cap \mathcal{Q}=\emptyset$ and $\iota\left(\mathcal{T}, x_{1}\right) \geq \Theta(\mathcal{Q}) \geq \theta_{0}$. Apply Lemma 5.2 to the walk $U_{1}=x_{0}, \alpha_{1}, x_{1}, \alpha_{2}, x_{2}$ and the half-neighborhood $\mathcal{T}$ to choose a walk $U_{2}$ from $x_{2}$ to $x_{0}$, let $\mathcal{D}$ be the corresponding closed disc, and set $\mathcal{Q}^{\prime}=\mathcal{Q} \cup \mathcal{D}$. (Figure 4 shows the described change of $W$. The geodesic walks $J_{1}, J_{2}, J_{3}$ are the boundary walks of $Q^{\prime}$ that will replace $W$.) It follows from (1) of Lemma 5.2 and the assumption that $\iota\left(\mathcal{T}, x_{1}\right)>0$ that no point in the interior of $\mathcal{D}$ may appear in a boundary walk of $\mathcal{Q}$. It follows easily from this that $\mathcal{Q}^{\prime}$ is also a front. Let $\mathcal{Q}^{\prime \prime}$ be the front obtained by taking the union of $\mathcal{Q}^{\prime}$ with every connected component of $\mathcal{R} \backslash \mathcal{Q}^{\prime}$ which has only finitely many vertices. It follows from (3) of Lemma 5.2 that $\ell\left(\mathcal{Q}^{\prime \prime}\right) \leq \ell\left(\mathcal{Q}^{\prime}\right) \leq \ell(\mathcal{Q})$. Further, if $\mathcal{Q}^{\prime \prime}$ is a $\left(s^{\prime \prime}, t^{\prime \prime}, u^{\prime \prime}\right)$-front, then $s^{\prime \prime} \leq s$, and it follows from the assumption that $\mathcal{Q}$ is full that $u^{\prime \prime} \geq u$. All of these conclusions imply that $\Theta\left(\mathcal{Q}^{\prime \prime}\right) \geq \Theta(\mathcal{Q}) \geq \theta_{0}$. The following equation also follows from this construction:

$$
w_{\zeta}(\mathcal{Q})-w_{\zeta}\left(\mathcal{Q}^{\prime \prime}\right) \geq w_{\zeta}(\mathcal{Q})-w_{\zeta}\left(\mathcal{Q}^{\prime}\right) \geq w_{\zeta}\left(U_{1}\right)-w_{\zeta}\left(U_{2}\right)
$$

If $\lambda\left(U_{2}\right)>\lambda\left(U_{1}\right)$, then by (3) of Lemma 5.2 we have $w_{\zeta}\left(U_{1}\right)-w_{\zeta}\left(U_{2}\right) \geq 1$, but this gives a contradiction to the choice of $\mathcal{Q}$. If $\lambda\left(U_{2}\right) \leq \lambda\left(U_{1}\right)$, then either $\lambda\left(U_{2}\right)=1$ 
or $\lambda\left(U_{2}\right)=2$. In the former case, it follows from Observation 2.2 and part (a) of Lemma 4.1 that $w_{\zeta}\left(U_{1}\right)-w_{\zeta}\left(U_{2}\right) \geq \frac{2 \nu}{\zeta}\left(1-\sin \frac{\pi-\theta_{0}}{2}\right)=1$, giving us a contradiction to the choice of $\mathcal{Q}$. In the latter case, the walk $U_{2}$ is of the form $x_{2}, \beta_{2}, y, \beta_{1}, x_{0}$, and we may choose a geodesic $\alpha^{\prime}$ from $x_{1}$ to $y$ which is contained in $\mathcal{D}$. Now by applying Observation 2.2 to the two discs contained in $\mathcal{D}$ and bounded by $x_{1}, \alpha^{\prime}, y, \beta_{1}, x_{0}, \alpha_{1}, x_{1}$ and $x_{1}, \alpha^{\prime}, y, \beta_{2}, x_{2}, \alpha_{2}, x_{1}$ and then using part (b) of Lemma 4.1 we again find that $w_{\zeta}\left(U_{1}\right)-w_{\zeta}\left(U_{2}\right) \geq \frac{2 \nu}{\zeta}\left(1-\sin \frac{\pi-\theta_{0}}{2}\right)=1$, contradicting the choice of $\mathcal{Q}$. This completes the proof.

It is also possible to prove the above lemma by minimizing the ordinary length of the front. This requires a slight strengthening of Lemma 4.1 to show that every time Lemma 5.2 is used to replace a walk $W$ with a new walk $W^{\prime}$, the length of $W^{\prime}$ is a bounded distance below the length of $W$.

If $G$ is a graph, $X \subseteq V(G)$ and $A_{1}, A_{2}$ are distinct ends of $G$, then we say that $X$ separates $A_{1}$ and $A_{2}$ if there exist distinct components $H_{1}, H_{2}$ of $G \backslash X$ so that every ray in $A_{i}$ has all but finitely many vertices in $H_{i}$ for $i=1,2$. If $\mathcal{R}$ is a polygonal metric space, $G$ is the graph $(V(\mathcal{R}), E(\mathcal{R})), \mathcal{T} \subseteq \mathcal{R}$, and $A$ is an end of $G$, then we say that $\mathcal{T}$ captures $A$ if every ray in $A$ has all but finitely many vertices in $\mathcal{T}$. Our next lemma shows the existence of fronts with certain useful properties.

Lemma 5.4. Let $\mathcal{R}$ be a polygonal surface, let $G$ be the graph $(V(\mathcal{R}), E(\mathcal{R}))$, let $Y$ be a finite set of vertices, let $\mathcal{E}$ be a finite subset of $\operatorname{Ends}(G)$, and let $\Xi$ be a finite collection of closed curves in $\mathcal{R}$. Then there exists a full front $\mathcal{Q}$ with the following properties:

(i) $Y \subseteq V^{i}(\mathcal{Q})$.

(ii) Every closed curve in $\Xi$ is contained in $\mathcal{Q}$.

(iii) Every end in $\mathcal{E}$ is captured by a component of $\mathcal{R} \backslash \mathcal{Q}$, and no two distinct ends from $\mathcal{E}$ are captured by the same component.

Proof. It follows from the definition of ends that we may choose a finite set $Z \subseteq$ $V(G)$ so that $Z$ separates every pair of ends in $\mathcal{E}$. Let $J$ denote the set of all faces which contain a point in $Y \cup Z$ or contain a point in some curve in $\Xi$. It follows from the fact that each closed curve is compact that $J$ is finite. Since $\mathcal{R}$ is connected, we may choose a finite set of faces $J^{\prime} \supseteq J$ so that $\mathcal{Q}^{\prime}=\bigcup J^{\prime}$ is connected. Now, by construction, $\mathcal{Q}^{\prime}$ is a front which satisfies (i)-(iii). Finally, let $\mathcal{Q}$ be the union of $\mathcal{Q}^{\prime}$ and all components of $\mathcal{R} \backslash \mathcal{Q}^{\prime}$ that contain only finitely many vertices. Since $\mathcal{Q}^{\prime}$ is the union of finitely many faces, $V^{i}(\mathcal{Q})$ is finite and $\mathcal{Q}$ is a full front satisfying (i)-(iii).

Proof of Theorem 1.3. Let $\kappa^{-}=\sum_{v \in V(\mathcal{R}): \kappa(v)<0} \kappa(v)$ and let $G$ be the graph $(V(\mathcal{R}), E(\mathcal{R}))$. If $G$ has infinitely many ends, then choose a finite $\mathcal{E} \subseteq \operatorname{Ends}(G)$ so that $\kappa^{-}-4 \pi+2 \pi|\mathcal{E}|>0$. Now, by applying Lemma 5.4 to $\mathcal{E}, Y=\emptyset$ and $\Xi=\emptyset$ we get a front which contradicts Lemma [5.3. Thus, $\operatorname{Ends}(G)$ is finite. Similarly, if the fundamental group of $\mathcal{R}$, hereafter denoted $\pi_{1}(\mathcal{R})$, is not finitely generated, then by choosing a sufficiently large subset $\Xi$ of a minimal generating set of curves for $\pi_{1}(\mathcal{R})$ and applying Lemma 5.4 and then Lemma 5.3 , we obtain a contradiction. Thus, we may choose a finite set $\Xi$ of closed curves in $\mathcal{R}$ which generate $\pi_{1}(\mathcal{R})$. By a similar argument, we find that $\sum_{v \in V(\mathcal{R})} \kappa(v)$ converges. 
Now, choose a finite set $Y \subseteq\{v \in V(\mathcal{R}): \kappa(v)>0\}$ so that

$$
\left\lceil\frac{1}{2 \pi} \sum_{v \in V(\mathcal{R})} \kappa(v)\right\rceil=\left\lceil\frac{1}{2 \pi} \kappa^{-}+\frac{1}{2 \pi} \sum_{y \in Y} \kappa(y)\right\rceil
$$

and apply Lemma 5.4 to $\mathcal{E}=\operatorname{Ends}(G), \Xi$, and $Y$ to obtain a full $(s, t, u)$-front $\mathcal{Q}$. Let $\mathcal{T}$ be a connected component of $\mathcal{R} \backslash \mathcal{Q}$. If there exist two boundary walks $W_{1}, W_{2}$ of $\mathcal{Q}$ which are both in the boundary of $\mathcal{T}$, we have a contradiction to the assumption that $\Xi$ generates $\pi_{1}(\mathcal{R})$. (In particular, it follows that $t=u$.) Thus, there is a unique boundary walk $W$ of $\mathcal{Q}$ which is in the boundary of $\mathcal{T}$. Modify $\mathcal{T}$ to form $\mathcal{T}^{\prime}$ by identifying it with a closed disc along the boundary walk $W$. If $\mathcal{T}^{\prime}$ is not homeomorphic to the plane, then we again have a contradiction to the assumption that $\Xi$ generates $\pi_{1}(\mathcal{R})$. Thus, $\mathcal{T}$ is homeomorphic to $S^{1} \times(0,1)$, and it follows that $\mathcal{R}$ is homeomorphic to a space obtained from a compact closed 2maninfold of Euler characteristic $s$ by removing a set of $t$ points. Furthermore, by Lemma 5.3 we have that $\sum_{y \in Y} \kappa(y)-2 \pi s+2 \pi t+\kappa^{-} \leq 0$, so it follows from our choice of $Y$ that $\sum_{v \in V(\mathcal{R})} \kappa(v)-2 \pi s+2 \pi t \leq 0$ as desired.

\section{HiguCHI's CONJECTURE}

In this section we shall apply the key lemma from the previous section to prove Theorem 1.7 (which implies Higuchi's conjecture). We begin with some additional terminology. Let $G$ be a plane graph with $\phi(v)>0$ for every $v \in V(G)$. If $a_{1}, \ldots, a_{k}$ is a list of positive integers with $a_{1} \leq \cdots \leq a_{k}$, then we say that a vertex $v$ has pattern $\left(a_{1}, \ldots, a_{k}\right)$ if the faces incident with $v$ may be ordered $f_{1}, \ldots, f_{k}$ so that $f_{i}$ has size $a_{i}$ for $1 \leq i \leq k$. The following observation is straightforward to verify.

Observation 6.1. If $v$ is a vertex of a 2-cell embedded graph $G$ with degree at least 3 and $\phi(v)>0$, then $v$ has one of the patterns in Table 1 .

Motivated by this observation, we define a face to be big if it has size $\geq 42$. The following lemma shows that distinct big faces cannot be too close, unless the graph is either a prism or an antiprism. Examples of graphs of a prism and an antiprism and their projective planar analogues are indicated in Figures 11 and 2 ,

Lemma 6.2. Let $G$ be a 2-cell embedded graph with minimum degree at least 3, all faces of finite size, and everywhere positive combinatorial curvature $\phi$. If uv is an edge of $G$ that is not incident with a big face but $u$ and $v$ are both incident with a big face, then $G$ is either a prism, an antiprism, a projective prism or a projective antiprism.

Proof. If $u$ or $v$ has pattern $(3, i, j)$ where $j \geq 42$, then the vertex of the facial triangle other than $u, v$ lies on two big faces, which is impossible. Thus, the pattern $(3, i, j)$ for $j \geq 42$ is not possible at $u$ and $v$. Consequently, according to Table1, $u, v$ either both have pattern $(4,4, j)$ or both have pattern $(3,3,3, j)$ for some $j \geq 42$. In the first case, choose $u^{\prime}, v^{\prime}$ so that $u u^{\prime} v^{\prime} v u$ is the facial walk of a 4 -face. Then both $u^{\prime}$ and $v^{\prime}$ have pattern $(4,4, j)$, and we may choose $u^{\prime \prime}, v^{\prime \prime}$ distinct from $u, v$ so that $u^{\prime} u^{\prime \prime} v^{\prime \prime} v^{\prime} u^{\prime}$ is a facial walk. Since this process may be repeated ad infinitum, the graph $G$ must be either a prism or a projective prism. Similarly, if $u, v$ both have pattern $(3,3,3, j)$, then we may choose a vertex $u^{\prime}$ so that $v, u, u^{\prime}$ lie on a triangular face and choose $v^{\prime}$ so that $v, v^{\prime}, u^{\prime}$ lie on a triangular face. Next, we may choose $u^{\prime \prime}$ distinct from $v$ so that $u^{\prime}, v^{\prime}, u^{\prime \prime}$ lie on a triangular face and choose $v^{\prime \prime}$ distinct 
TABLE 1. Possible patterns in positively curved graphs

\begin{tabular}{|l|l|l|}
\hline Pattern of $v$ & Parameters & $\phi(v)$ \\
\hline$(3,3,3,3, j)$ & $3 \leq j \leq 5$ & $\frac{1}{j}-\frac{1}{6}$ \\
$(3,3,3, j)$ & $3 \leq j$ & $\frac{1}{j}$ \\
$(3,3,4, j)$ & $4 \leq j \leq 11$ & $\frac{1}{j}-\frac{1}{12}$ \\
$(3,3,5, j)$ & $5 \leq j \leq 7$ & $\frac{1}{j}-\frac{2}{15}$ \\
$(3,4,4, j)$ & $4 \leq j \leq 5$ & $\frac{1}{j}-\frac{1}{6}$ \\
$(3, i, j)$ & $3 \leq i \leq 6$ and $i \leq j$ & $\frac{1}{i}+\frac{1}{j}-\frac{1}{6}$ \\
$(3,7, j)$ & $7 \leq j \leq 41$ & $\frac{1}{j}-\frac{1}{42}$ \\
$(3,8, j)$ & $8 \leq j \leq 23$ & $\frac{1}{j}-\frac{1}{24}$ \\
$(3,9, j)$ & $9 \leq j \leq 17$ & $\frac{1}{j}-\frac{1}{18}$ \\
$(3,10, j)$ & $10 \leq j \leq 14$ & $\frac{1}{j}-\frac{1}{15}$ \\
$(3,11, j)$ & $11 \leq j \leq 13$ & $\frac{1}{j}-\frac{5}{66}$ \\
$(4,4, j)$ & $4 \leq j$ & $\frac{1}{j}$ \\
$(4,5, j)$ & $5 \leq j \leq 19$ & $\frac{1}{j}-\frac{1}{20}$ \\
$(4,6, j)$ & $6 \leq j \leq 11$ & $\frac{1}{j}-\frac{1}{12}$ \\
$(4,7, j)$ & $7 \leq j \leq 9$ & $\frac{1}{j}-\frac{3}{28}$ \\
$(5,5, j)$ & $5 \leq j \leq 9$ & $\frac{1}{j}-\frac{1}{10}$ \\
$(5,6, j)$ & $6 \leq j \leq 7$ & $\frac{1}{j}-\frac{2}{15}$ \\
\hline
\end{tabular}

from $u^{\prime}$ so that $v^{\prime}, u^{\prime \prime}, v^{\prime \prime}$ lie on a triangular face. As before, this process may be repeated ad infinitum, and it follows that $G$ must be an antiprism or a projective antiprism.

For any subset $X \subseteq V(G)$, we let $N(X)$ denote the set of vertices adjacent to a vertex in $X$. The following lemma gives a lower bound on the sum of $\phi(x)$ over a set of the form $X \cup N(X)$.

Lemma 6.3. If $G$ is neither an ordinary nor projective prism or antiprism, and $\phi$ is everywhere positive, then $\sum_{x \in X \cup N(X)} \phi(x) \geq \frac{|X|}{1722}$ for every $X \subseteq V(G)$.

Proof. Call a vertex bad if it is incident with a big face, and good otherwise. Starting with the weight function $\phi$, we create a new function $\phi^{\prime}$ by the following discharging rule. If $v$ is a good vertex of degree $d$, send $\frac{\phi(v)}{d+1}$ to each bad neighbor of $v$. Suppose that $v$ is a good vertex which is adjacent to a bad vertex. Then $v$ must either be incident with two squares, or with one triangle and another face of size $\leq 6$. An easy check of Table 1 shows that this implies $\phi(v) \geq \frac{1}{132}$. Therefore, $\phi^{\prime}(v) \geq \frac{1}{132(d+1)} \geq \frac{1}{792}$. The same inequality holds for the modified curvature of every bad vertex in $X$ since it receives such a value from some good neighbor by Lemma 6.2. It follows from this that $\phi^{\prime}(w) \geq \frac{1}{1722}$ for every vertex $w$. Thus, $\sum_{x \in X \cup N(X)} \phi(x) \geq \sum_{x \in X} \phi^{\prime}(x) \geq \frac{|X|}{1722}$ as desired. 
With this, we are ready to prove the theorem.

Proof of Theorem 1.7. Let $G$ be a 2-cell embedded graph with minimum degree $\geq 3$ and every face of finite size. Suppose that $G$ is neither a prism nor an antiprism (planar or projective planar) and that $\phi$ is everywhere positive. We form a polygonal metric space $\mathcal{R}$ by associating each face in $G$ of size $k$ with a regular polygon in the plane having $k$-sides of length one. Note that by this construction, $\nu=1$, and $\kappa(v)=2 \pi \phi(v)$ for every vertex $v$. Now, it follows from Lemma 6.3 and Theorem 1.3 that $G$ is finite. By the Descartes-Euler formula (Theorem 1.1) we have that $\sum_{v \in V(G)} \phi(v)=2$, so by applying Lemma 6.3 to $V(G)$, we deduce that $|V(G)| \leq$ 3444 as claimed.

\section{ACKNOWLEDGEMENT}

We thank Paul Seymour and Ken Kawarabayashi for several helpful discussions on the subject of this paper.

\section{REFERENCES}

1. A. D. Aleksandrov, V. A. Zalgaller, Intrinsic Geometry of Surfaces, Amer. Math. Soc., 1967. MR.0216434 (35:7267)

2. http://www.ams.org/new-in-math/cover/descartes1.html

3. M. Gromov, Hyperbolic groups, in "Essays in Group Theory," S. M. Gersten (Editor), M.S.R.I. Publ. 8, Springer, 1987, pp. 75-263. MR0919829 (89e:20070)

4. Y. Higuchi, Combinatorial curvature for planar graphs, J. Graph Theory 38 (2001) 220-229. MR.1864922 (2002i:05109)

5. J. Milnor, A note on curvature and fundamental group, J. Differential Geometry 2 (1968) 1-7. MR.0232311 (38:636)

6. B. Mohar, Embeddings of infinite graphs, J. Combin. Theory, Ser. B 44 (1988) 29-43. MR0923264 (88k:05074)

7. S. B. Myers, Riemannian manifolds with positive mean curvature, Duke Math. J. 8 (1941) 401-404. MR0004518 (3:18f)

8. T. Réti, E. Bitay, Z. Kosztolányi, On the polyhedral graphs with positive combinatorial curvature, Acta Polytechnica Hungarica 2 (2005) 19-37.

9. D. A. Stone, A combinatorial analogue of a theorem of Myers, Illinois J. Math. 20 (1976) 12-21. MR0410602 (53:14350a)

10. D. A. Stone, Correction to my paper: "A combinatorial analogue of a theorem of Myers", Illinois J. Math. 20 (1976) 551-554. MR0410603 (53:14350b)

11. L. Sun, X. Yu, Positively curved cubic plane graphs are finite, J. Graph Theory 47 (2004) 241-274. MR2096789 (2006a:05047)

12. W. Woess, A note on tilings and strong isoperimetric inequality, Math. Proc. Camb. Phil. Soc. 124 (1998) 385-393. MR1636552 (99f:52026)

Department of Mathematics, Simon Fraser University, Burnaby, British Columbia, V5A 1S6, CANADA

E-mail address: mdevos@sfu.ca

Department of Mathematics, University of Ljubljana, 1000 Ljubljana, Slovenia

Current address: Department of Mathematics, Simon Fraser University, Burnaby, British Columbia, V5A 1S6, Canada

E-mail address: bojan.mohar@fmf.uni-lj.si 